\title{
Atopic Dermatitis as a Paradoxical Effect of Secukinumab for the Treatment of Psoriasis
}

\author{
Eduardo V. Mendes Roncada Victoria Romanini Brambilla \\ Beatriz Freitas Filitto Mariana Pirajá Genta \\ Marilda Ap. M. Morgado de Abreu \\ Departament of Dermatology, UNOESTE - Universidade do Oeste Paulista, Hospital \\ Regional de Presidente Prudente, São Paulo, Brazil
}

\section{Keywords}

Atopic dermatitis $\cdot$ Paradoxical effect $\cdot$ Psoriasis $\cdot$ Secukinumab

\begin{abstract}
In the therapeutic arsenal to treat moderate to severe psoriasis, the new agents are secukinumab and ustekinumab, which are fully human monoclonal antibodies, directed against IL-17A and IL-12/23, respectively, which have been shown to be effective and safe in several studies. Their side effects are rare, and the most frequently reported side effects were infection, especially nasopharyngitis, headache, pruritus, high blood pressure, and low back pain. Unlike the side effects, the paradoxical reaction can be defined by the appearance or exacerbation of a pathological condition that usually responds to a certain class of drug. The appearance of this reaction in patients using anti-interleukins is poorly described; however, as they are new drugs, they may be more common than the literature reports. We describe a case of a paradoxical reaction, with the appearance of atopic dermatitis, after using secukinumab to treat psoriasis.
\end{abstract}

(C) 2021 The Author(s).

Published by S. Karger AG, Basel

\section{Introduction}

Psoriasis is a chronic immune-mediated inflammatory disease whose therapeutic alternative is the use of anti-interleukin 17-A agents, such as secukinumab, which have been proved to be effective and safe in several studies. Paradoxical reactions to this class of antibodies have been poorly described, since they are relatively recent drugs; they may be more common than those reported in the literature $[1,2]$. We describe a case of a paradoxical reaction of atopic dermatitis, after using secukinumab to treat severe psoriasis. 


\section{Case Report/Case Presentation}

The case is a 59-year-old Caucasian woman, with vulvar, nail and inverse psoriasis (intergluteal, inframammary, and infra-abdominal regions) for 8 years. She was previously hypertensive, diabetic, dyslipidemic, and depressive and had cardiac disease.

Secukinumab was started on at a dose of $300 \mathrm{mg}$, via SC, for 5 consecutive weeks, followed by monthly application. There was an initial improvement in the induction phase, but after 7 days of the sixth dose, it opened acute symmetrical eczema, located in the antecubital and popliteal folds, accompanied by intense itching, places that have never been affected by inverted psoriasis before, associated with UVB-NB phototherapy without improvement. It evolved with secondary bacterial infection, characterized by exulcerations, fissures, and purulent secretion on the eczema areas, progressing to progressive worsening of psoriatic plaques and even the development of exfoliative erythroderma.

A skin biopsy of an antecubital and popliteal fold lesion was performed, showing histopathological examination compatible with atopic dermatitis; the antinuclear antibody test (ANA-HEp-2) and HTLV-I/II were negative (Fig. 1). There was no other known trigger factor, such as trauma, surgery, stress, introduction of new drugs, or systemic corticosteroid use, which would justify the precipitation of such exacerbation, except for the secondary infection of eczema.

Atopic dermatitis was diagnosed as a paradoxical event to the use of secukinumab. We opted for patient hospitalization, suspension of secukinumab, and rescue therapy with cyclosporine, $5 \mathrm{mg} / \mathrm{kg} /$ dose; intravenous antibiotic therapy, and skin barrier restorative creams and topical corticosteroids were started. The patient evolved with significant improvement in atopic dermatitis and psoriasis, presenting PASI 75 at week 6 of cyclosporine, without recurrence of atopic dermatitis.

\section{Discussion/Conclusion}

The FEATURE study also showed the appearance of mild to moderate infections after secukinumab, with cases of candidiasis and reactions at the application sites that did not lead to treatment discontinuation $[1,3]$. In the case reported here, the patient developed atopic dermatitis after the introduction of secukinumab. This effect may be due to an imbalance in Th1 and Th2 pathways. In psoriasis, there is an exacerbation of Th1 and Th17 activity and, in atopic eczema, the predominance of Th2. Thus, the inhibition of the Th1/Th17 pathway by secukinumab may have led to an exacerbation of the Th2 pathway and the consequent appearance of atopic eczema in the patient described [1,4].

Recently, Burlando et al. [1] reported a similar case, with the appearance of blepharitis, cheilitis, and nasal dermatitis, 4 days after each injection of secukinumab, with the clinical diagnosis of atopic dermatitis being made, and the spontaneous disappearance of the reaction after discontinuing the drug. In our patient, we observed the appearance of atopic dermatitis the day after the drug was administered, and in a different way, the suspension of secukinumab was not sufficient for the remission of eczema, and it was necessary to introduce cyclosporine to control the disease.

In another study, Ishiuji et al. [5] described 2 cases of atopic dermatitis as a paradoxical effect to the use of ustekinumab for the treatment of psoriasis. In this study, the authors propose the participation of immunoglobulin $\mathrm{E}$ in the pathophysiology of the paradoxical reaction.

In the case described, we do not have the immunoglobulin $\mathrm{E}$ dosages prior to the onset of atopic dermatitis; however, the dosages performed after the introduction of cyclosporine

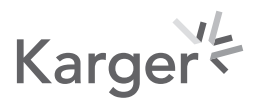


Fig. 1. Antecubital region with atopic dermatitis.

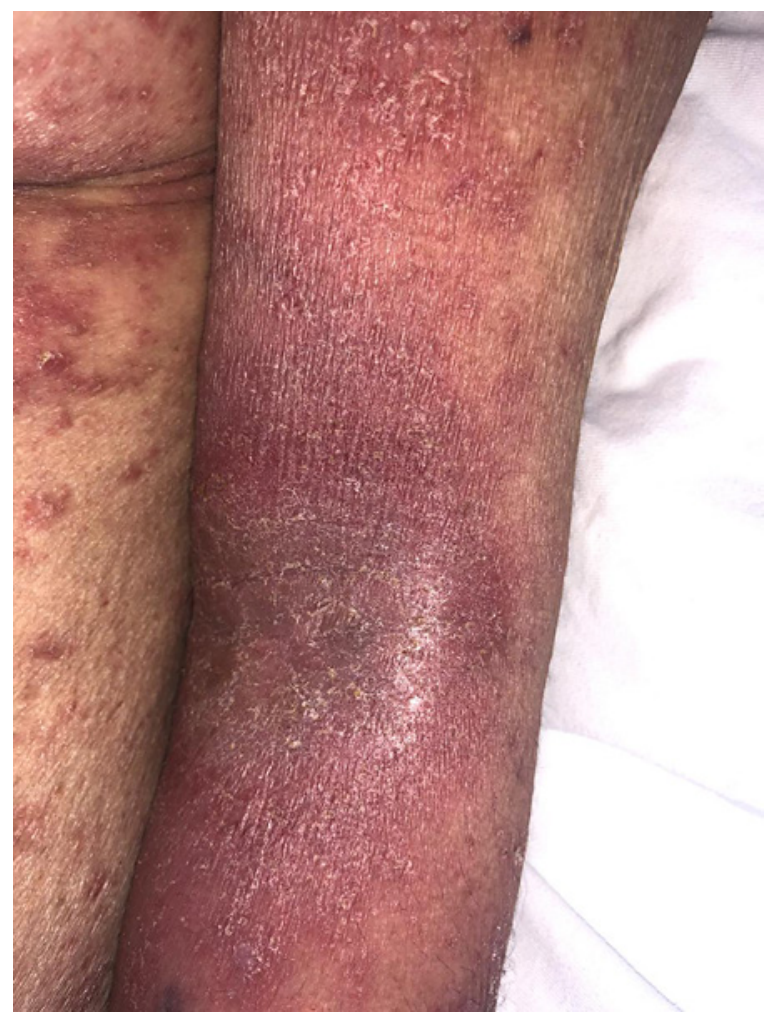

were increased. Therefore, we consider it prudent to always carry out an accurate assessment of the current or past history of atopic dermatitis before the introduction of an anti-interleukin immunobiological agent, since the association described may be more common than the literature shows. Therefore, further studies are needed so that we can estimate the real prevalence and unveil the pathophysiological mechanisms of this serious paradoxical event.

\section{Acknowledgement}

The authors thank the Regional Hospital of Presidente Prudente for their support.

\section{Statement of Ethics}

The patient gave consent for publication. The research was conducted ethically in accordance with the World Medical Association Declaration of Helsinki. Written informed consent was obtained from the patient for publication of this case report and any accompanying images.

\section{Conflict of Interest Statement}

The authors have no conflicts of interest to declare.

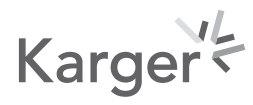




\section{Funding Sources}

The authors declare that there was no funding.

\section{Author Contributions}

V.R. Brambilla treated the patient and wrote the case report, M.M. Morgado evaluated the data, E.M. Roncada performed the visits and treated the patient, and M.P. Genta also visited the patient and wrote the case report. B. Freitas Filitto also evaluated the data and wrote the case report.

\section{References}

1 Burlando M, Cozzani E, Russo R, Parodi A. Atopic-like dermatitis after secukinumab injection: a case report. Dermatol Ther. 2019 Jan;32(1):e12751.

2 Langley RG, Elewski BE, Lebwohl M, Reich K, Griffiths CEM, Papp K, et al. Secukinumab in plaque psoriasis: results of two phase 3 trials. Br J Dermatol. 2015 Feb;172(2):484-93. Epub 2014 Dec 114.

3 Blauvelt A, Prinz JC, Gottlieb AB, Kingo K, Sofen H, Ruer-Mulard M, et al. Secukinumab administration by prefilled syringe: efficacy, safety and usability results from a randomized controlled trial in psoriasis (FEATURE). Br J Dermatol. 2015;172(2):484-93.

4 Eyerich S, Onken AT, Weidinger S, Franke A, Nasorri F, Pennino D, et al. Mutual antagonism of T cells causing psoriasis and atopic eczema. N Engl J Med. 2011 Jul;365:231.

5 Ishiuji Y, Umezawa Y, Asahina A, Fukuta H, Aizawa N, Yanaba K, et al. Exacerbation of atopic dermatitis symptoms by ustekinumab in psoriatic patients with elevated serum immunoglobulin E levels: report of two cases. J Dermatol. 2018 Jun;45(6):732-4. Epub 2018 Mar 22. 